\title{
Il coordinamento della pianificazione sul territorio nell'ordinamento italiano
}

\author{
Fabio Merusi \\ Catedrático de Derecho Administrativo \\ Universidad de Pisa
}

\begin{abstract}
SOMMARIO: 1. LA PROGRAMMAZIONE GERARCHICA DEL 1942. I PIANI TERRITORIALI DI COORDINAMENTO. NON FURONO MAI REALIZZATI, TRANNE CHE IN QUALCHE ZONA INDUSTRIALE. 2. LE PROGRAMMAZIONI REGIONALI. 3. LA RIFORMA DEGLI ANNI '90 DEL SECOLO SCORSO. NUOVE AUTONOMIE AI COMUNI. IL PIANO TERRITORIALE DI COORDINAMENTO DEI PIANI COMUNALI VIENE AFFIDATO ALLA PROVINCIA. 4. RIFORMA DEL TITOLO V DELLA COSTITUZIONE NEL 2001: TUTTI GLI ENTI TERRITORIALI, STATO, REGIONI, CITTÀ METROPOLITANE, PROVINCE E COMUNI SONO SULLO STESSO PIANO, DI CONSEGUENZA TUTTI DEBBONO PARTECIPARE AL PROCEDIMENTO DI ELABORAZIONE DEI PIANI. COORDINAMENTO NEI PROCEDIMENTI DI PIANIFICAZIONE DI TUTTE LE PIANIFICAZIONI SETTORIALI CHE INTERESSANO IL TERRITORIO. LA NUOVA COMPETENZA LEGISLATIVA ATTRIBUITA ALLE REGIONI SUL GOVERNO DEL TERRITORIO DETERMINA LA POSSIBILITÀ DI DISCIPLINE DIVERSE IN OGNI SINGOLA REGIONE ANCHE SULLE PIANIFICAZIONI DI AREA VASTA ATTRIBUITE ALLE PROVINCE. 5. CONCLUSIONI RIASSUNTIVE SULLA DISCIPLINA ATTUALE DELLA PIANIFICAZIONE DI AREA VASTA.
\end{abstract}

1. Nell'ordinamento italiano si può cominciare a parlare di pianificazione di area vasta, cioè di livello superiore ai comuni, già nella legge sull'espropriazione per pubblica utilità del 1865, laddove all'interno della complessa disciplina espropriativa conteneva norme riguardanti la materia urbanistica ed, in particolare, la pianificazione del territorio. Tuttavia è soltanto con la legge urbanistica del 1942 che la pianificazione sovracomunale assume una fisionomia autonoma e connotati di sicuro rilievo giuridico, dal momento che attraverso tale strumento il legislatore introduce il sistema della pianificazione territoriale che, in virtù della suddivisione del territorio per zone omogenee, tutte corrispondenti ad un determinato tipo di piano ordinato secondo un sistema gerarchico che procede dall'alto verso il basso, finisce per sottoporre alla disciplina urbanistica ogni aspetto del territorio anche se non strettamente collegato alla materia urbanistica propriamente detta.

L'impianto della legge del '42 appare fortemente caratterizzato da alcuni principi generali che ne regolano l'impianto, corrispondenti all'ideologia 
gerarchica e autoritaria tipica dell'epoca anche in altri settori. In primis l'impostazione "totalitaria": la pianificazione urbanistica è riferita nelle sue articolazioni all'intero territorio nazionale.

Questo impianto di così generale portata si articola secondo un ferreo principio gerarchico secondo cui le varie tipologie di piani ivi previste si susseguono procedendo dal generale al particolare, in modo che il piano di grado inferiore operi soltanto specificando le previsioni già contemplate nel piano di grado superiore, così da conformarsi ad esse.

La gerarchia e la conformità dell'autorità pianificante inferiore rispetto alle regole predisposte dall'autorità pianificante maggiore trasportano in ambito urbanistico una più generale concezione dei rapporto fra lo Stato e gli enti locali in vigore all'epoca di riferimento.

Infatti sono attribuiti, secondo un principio gerarchico che procede dall'alto verso il basso, rispettivamente allo Stato, poi alla Regione, infine ai Comuni i vari poteri pianificatori, i quali, però, man mano che si scende nella scala gerarchica divengono sempre meno autonomi e meno indipendenti.

Lo Stato e il suo apparato sono gli organi che regolano l'intero procedimento pianificatorio sul territorio nazionale e lo fanno sia in modo diretto -vale a dire attraverso la predisposizione di vincoli operanti nei confronti dei soggetti di pianificazione inferiore ed attraverso l'impiego del potere di direttiva-, sia in modo indiretto, vale a dire attraverso l'inserimento nei processi di pianificazione minore in virtù del meccanismo delle intese oppure attraverso poteri di controllo che si manifestano in particolare in atti di approvazione attraverso i quali la volontà del controllante si sovrappone a quella del controllato.

Un simile assetto sottintende una concezione organizzatoria dei rapporti fra Stato ed enti locali dove il primo opera in posizione di superiorità gerarchica rispetto ai secondi.

Si pensi che una circolare del Ministero dell'Interno del 21 agosto 1939, n. 60-3 definiva i comuni "enti ausiliari" dello Stato.

All'interno di questo quadro normativo viene prevista anche una disciplina per la pianificazione di area vasta: la legge urbanistica, infatti, regola l'istituto del piano territoriale di coordinamento. 
Il suddetto piano sovracomunale, previsto come facoltativo, era di competenza statale e si basava sulla tecnica dell'azzonamento per aree omogenee.

In teoria avrebbe dovuto avere la funzione di coordinamento e di orientamento dell'attività urbanistica comunale. I piani di coordinamento non avevano efficacia diretta sulla disciplina del territorio, ma solo indiretta in quanto avevano come destinatari i comuni.

In pratica non furono mai realizzati, tranne in qualche caso assai limitato nel quale veniva in gioco la disciplina di qualche insediamento industriale.

Solo nel secondo dopoguerra se ne fece una numerosa applicazione per un intervento speciale: l'industrializzazione del Mezzogiorno.

2. L'assetto sommariamente delineato muta con l'entrata in vigore della Carta Costituzionale, con l'istituzione delle Regioni e, di conseguenza, col trasferimento di funzioni amministrative a queste ultime anche in materia urbanistica.

A seguito di tali cambiamenti risulta variata la logica applicativa della legge urbanistica del 1942 ancorché questa rimanga formalmente in vigore. In primis vengono creati nuovi soggetti di pianificazione coi quali vengono attribuite funzioni e compiti propri, con conseguente riduzione del ruolo e della funzione dello Stato.

Sul piano soggettivo, quindi, si assiste ad una scissione che vede afferire gli interessi di carattere locale alla potestà pianificatoria delle Regioni, mentre gli interessi di carattere sovraregionale e generale alla potestà statale, secondo un principio di equiordinazione delle relazioni regolato da confronti procedimentali e non più da imposizioni gerarchiche.

Al livello inferiore, invece, si ripropone lo schema gerarchico che aveva caratterizzato le relazioni fra Stato ed enti locali prima della Costituzione: al di fuori dello Stato e della Regione, gli altri soggetti pianificatori sono sottoposti al rispetto della gerarchia da essi imposta. Come dire che nella gerarchia la Regione si sostituisce allo Stato.

Anche la disciplina dell'area vasta sovracomunale subisce una modifica, ma, analogamente al passato, la nuova normativa non ha molta fortuna nella pratica. 
$\mathrm{Al}$ piano territoriale di coordinamento viene sostituito il piano territoriale regionale; esso -almeno in teoria- avrebbe dovuto produrre effetti, in parte di tipo obbligatorio, in parte di tipo reale, cioè in parte di disciplina diretta del territorio e in parte di vincolo nei confronti dei piani comunali.

I primi consentono alle direttive contenute nel piano di area vasta di costituire obblighi nei confronti dei titolari inferiori del potere di pianificazione, ma non nei confronti dello Stato; i secondi consentono al piano di area vasta di incidere direttamente su beni immobili dei privati per fini localizzativi o conservativi, limitando il potere pianificatorio del Comune, il quale potrà solo conformarsi a quanto da esso previsto.

L'efficacia diretta delle prescrizioni suddette sui beni del privato indicano il superamento della valenza interna dei piani territoriali di area vasta.

3. La pianificazione di area vasta subisce nuovamente rilevanti modificazioni con le riforme introdotte nell'ordinamento italiano degli anni ' 90.

In tale periodo, infatti, la legge sulle autonomie locali e la normativa, sulla semplificazione amministrativa e sul riparto di funzioni amministrative fra $\mathrm{i}$ vari enti locali territoriali, introducono nuovi principi entro cui ridisegnare anche la pianificazione territoriale.

La riforma delle autonomie locali delinea in senso autonomistico la figura del Comune e trasferisce ad esso funzioni un tempo qualificabili come statali.

In virtù del c.d. principio di sussidiarietà, secondo il quale alla miglior cura dell'interesse pubblico provvede il soggetto che più è vicino alle esigenze della popolazione insistente su un determinato territorio, il Comune risulta essere l'ente esponenziale maggiormente rappresentativo della collettività territoriale di riferimento con la conseguenza che proprio il Comune diventa la figura centrale per la cura degli interessi propri di una determinata realtà territoriale.

Ne deriva che il Comune deve poter operare con funzioni proprie ed esclusive ed in autonomia rispetto allo Stato ed alla Regione.

A fianco ad esso ulteriori soggetti, differenti da Stato e Regione, ma rappresentativi di collettività locali e funzionalmente vicine alla cura degli interessi dei soggetti interessati, si trovano a gestire l'interesse pubblico (e l'assetto del territorio), operando non su un piano di gerarchia, ma su un 
piano di equiordinazione, dove si provvede alla cura degli interessi sulla base di un confronto procedimentale.

Viene introdotto il concetto di gestione autonoma degli interessi da parte di più amministrazioni: si passa da un sistema "integrato di attribuzione delle funzioni" ad un sistema pluralista dove diverse amministrazioni, ciascuna riferibile ad una determinata collettività, operano in maniera autonoma.

Conseguentemente un nuovo ruolo assume anche il coordinamento, onde evitare che vi siano contrasti ed incompatibilità fra le varie amministrazioni: si passa così dal controllo gerarchico al coordinamento finalizzato alla composizione di interessi pubblici.

Ma la vera novità della riforma degli enti locali del 1990 è data dalla comparsa di un nuovo ente regolatore, la Provincia, alla quale viene affidato il compito di coordinare la pianificazione comunale. Ad un piano di coordinamento che, nella versione originaria della legge del 1942, doveva riferirsi a zone territorialmente omogenee o alla soluzione emersa dopo l'istituzione delle Regioni che vedeva nelle Regioni anche il soggetto al quale affidare il coordinamento dei piani comunali, si sostituisce un ente istituzionale intermedio fra la Regione e il Comune, rivitalizzando così un ente, la Provincia, che per le sue limitate funzioni sembrava destinato a scomparire.

Altra rilevante novità istituzionale riferita alla pianificazione di un'area vasta è l'introduzione delle c.d. città metropolitane in cui la pianificazione viene riferita all'area di grandi città che trascende l'ambito strettamente comunale, ma non coincide necessariamente con la Provincia.

4. Questo quadro si perfeziona, ma allo stesso tempo si complica, con la modificazione del Titolo V della Costituzione intervenuta nel 2001 sul riparto di competenze fra lo Stato e le autonomie locali. Il nuovo articolo 118 della Costituzione mette sullo stesso piano gli enti territoriali, lo Stato, le città metropolitane, le Province ed i Comuni, il che legittima e consolida la soluzione già emersa dopo le riforme del 1990 secondo le quali le programmazioni dei vari enti non debbano avvenire a cascata imponendo gli enti più grandi vincoli agli enti progressivamente più piccoli le loro soluzioni, bensì attraverso il metodo della definizione procedimentale del piano da parte di tutti i soggetti interessati (lo Stato attraverso accordi con le Regioni, le Regioni attraverso procedimenti partecipati dalle Province e dai Comuni, la Provincia attraverso la partecipazione dei Comuni interessati e i Comuni 
attraverso la partecipazione di tutti i portatori di interessi presenti sul loro territorio). Mentre l'articolo 117, pure mutato dalla riforma del 2001, prevede una competenza concorrente fra Stato e Regioni nella materia governo del territorio che, sostituendosi all'urbanistica, legittima costituzionalmente la tendenza già in atto a concentrare nei piani di coordinamento di area vasta, cioè nella programmazione regionale e in quella delle Province, la disciplina di ogni programmazione settoriale riferita ad un determinato territorio.

Il quadro si complica perché la competenza concorrente fra lo Stato e le Regioni in materia di governo del territorio legittima l'emanazione in ogni Regione di differenti leggi di disciplina del territorio e conseguentemente di situazioni differenziate in merito al contenuto dei piani di area vasta e dei loro possibili effetti, se diretti almeno con riferimento ad alcuni oggetti di pianificazione, oppure soltanto indiretti nell'ambito del coordinamento dei piani comunali. Ogni Regione ha pertanto le sue varianti (o non ne ha alcuna se non ha legiferato), con la conseguenza che si possono indicare solo delle linee di tendenza che si riducono nell'identificare nella Provincia il soggetto pianificatore più o meno intenso dell'area vasta e nel "territorio" il presupposto legittimante il concentramento in un unico piano di tutti i piani di settore, che sopravvivono come tali nel coordinamento operato dalla Provincia, oppure si "confondono" interamente nel governo del territorio provinciale, a seconda delle soluzioni adottate dalle singole Regioni (per una delle soluzioni più recenti cfr., ad esempio, la legge regionale toscana n. 1 del 2005).

Da segnalare infine la recentissima soluzione da parte del D.Lgs. 2006, n. 157 in materia di beni culturali, il quale, in attuazione di quanto previsto dal nuovo art. 118 Cost., tenta di risolvere i rapporti fra il governo del territorio e una pianificazione che è andata sempre in parallelo con quella urbanistica: la disciplina del paesaggio, concepito quest'ultimo come un bene culturale e come tale da sempre sottoposto a vincoli statali. Secondo la nuova soluzione i piani paesaggistici debbono essere determinati in collaborazione fra lo Stato e le singole Regioni e di poi riflettersi in maniera cogente sulle pianificazioni inferiori, quella provinciale e quella comunale. In questo modo dovrebbero essere definitivamente ricomposti tutti i possibili aspetti del "governo del territorio".

Così almeno in teoria. Nella realtà il metodo procedimentale paritario fra tutti gli enti territoriali nella determinazione del piano provoca notevoli difficoltà nei tempi "di decisione" e soprattutto problemi insolubili quando esiste l'opposizione di interessi locali all'insediamento di strutture non gradite 
o che, più semplicemente, si vorrebbero imporre ad altri. I casi della localizzazione delle discariche dei rifiuti o delle stazioni di pompaggio di gas liquefatto e della realizzazione di autostrade o di linee ferroviarie ad alta velocità sono, credo, universalmente noti in quanto spesso e volentieri all'onore delle cronache.

5. Conclusioni. In Italia la programmazione di area vasta è attribuita alle Province o alle città metropolitane. La riforma costituzionale del 2001 sulla pari dignità degli enti territoriali comporta la partecipazione di tutti gli enti interessati al procedimento di determinazione di tutti i piani che possano interessarli. Non solo, ma l'attribuzione alle Regioni della legislazione concorrente nella materia "governo del territorio" prevista nella stessa riforma costituzionale rende ora possibile coordinare con la pianificazione urbanistica anche le varie pianificazioni di settore (paesaggio, vincoli idrogeologici e simili).

L'esperienza dimostra che l'ampia partecipazione ai procedimenti di pianificazione provoca lentezze nella elaborazione del piano e in numerosi casi la pratica impossibilità di prendere decisioni "difficili": è sintomatico in proposito che le leggi regionali più recenti prevedono procedure per superare i contrasti e giungere ad una decisione.

A ciò aggiungasi che l'attribuzione di una competenza legislativa concorrente alle Regioni determina la possibilità (in qualche caso già realizzata) che fra una Regione e l'altra ci siano delle differenze anche notevoli nella disciplina del procedimento di elaborazione e nel contenuto dei piani regionali e provinciali 